\title{
Theoretical Review of Mössbauer Effect, Hyperfine Interactions Parameters and the Valence Fluctuations in Eu Systems
}

\author{
Mahmoud Abdelrazek Ahmida \\ Electrical Engineering Department, The Higher Institute of Sciences and Technology, Ajdabiya, Libya \\ Email: Mahmida@yahoo.com
}

How to cite this paper: Ahmida, M.A. (2019) Theoretical Review of Mössbauer Effect, Hyperfine Interactions Parameters and the Valence Fluctuations in Eu Systems. Journal of Applied Mathematics and Physics, 7, 254-270.

https://doi.org/10.4236/jamp.2019.71021

Received: October 25, 2018

Accepted: January 28, 2019

Published: January 31, 2019

Copyright $\odot 2019$ by author(s) and Scientific Research Publishing Inc. This work is licensed under the Creative Commons Attribution International License (CC BY 4.0).

http://creativecommons.org/licenses/by/4.0/

\begin{abstract}
In this paper, an overview of the theory of Mössbauer effect is covered, and the main hyperfine interactions parameters which affect the shape of the resultant Mössbauer spectrum are explained and illustrated as well. In principle, Mössbauer effect applies to any and all nuclides, but in practice, certain ideal properties are desirable; that is, the conditions for recoil-free emission and absorption of gamma rays must be optimized. Therefore, briefly discussed in this review, one of the most commonly used for practical and fundamental studies the ${ }^{151} \mathrm{Eu}$ Mössbauer isotope. Also, the intermediate valence phenomena and their theoretical treatments are briefly discussed.
\end{abstract}

\section{Keywords}

Mössbauer Spectroscopy, Resonant Absorption, Recoil-Free Fraction, ${ }^{151} \mathrm{Eu}$ Mössbauer Isotope, Hyperfine Interactions, Valence Fluctuations

\section{Introduction}

Mössbauer spectroscopy is a method to measure small shifts and splittings with high accuracy in nuclear energy levels caused by interactions between the electrons and the nucleus. This method is based on the Mössbauer effect [1]-[7] which was discovered in 1958 by the German Physicist Rudolph L. Mössbauer and awarded the Nobel Prize in Physics for his discovery in 1961. The effect involves the emission and the absorption of $\gamma$-rays without recoil in solids. The issue of this technique is to present a means of measuring some of the comparatively weak interactions between the nucleus and the surrounding electrons using the Mössbauer spectroscopy. It can be used to provide information in many areas of science such as Physics, Chemistry, Biology and Metallurgy. It can give 
very precise information about the electronic and magnetic properties of the studied material. In the following sections, the Mössbauer effect is described using one of the most used Mössbauer isotopes among rare earth elements ${ }^{151} \mathrm{Eu}$ and a short explanation is given on the hyperfine interactions which determine the structure in a Mössbauer spectrum.

\subsection{Recoilless Emission and Absorption of $\gamma$-Rays}

Mössbauer showed that nuclear radiation can be emitted and absorbed by another nucleus of the same kind as the emitter without recoil if the nucleus placed in a solid [1]. When a free nucleus emits a $\gamma$-ray $\left(E_{\gamma}\right)$ during transition from an excited state level with energy $\left(E_{e}\right)$ to the ground state level with energy $\left(E_{g}\right)$ it will recoil. This recoil energy and velocity are due to conservation of energy and momentum, resulting in the emitted $\gamma$-ray being of lower energy than the nuclear transition energy $\left(E_{0}=E_{e}-E_{g}\right)$. The same is observed where the absorbing nucleus recoils, meaning the energy of the resonantly absorbed photon is less than the transition energy. To understand this effect [7], assume a free nucleus of mass $M$ with an excited state level at an energy $E_{0}$ and moving with a velocity $V$ along the direction of the emission of $E_{\gamma}$ energy (Figure 1).

The energy above the ground state at rest is $E_{0}+(1 / 2) M V^{2}$. When a $\gamma$-ray of energy $E_{\gamma}$ is emitted, the nucleus recoils and has a velocity $(V+v)$ and a total energy of $E_{0}+(1 / 2) M(V+v)^{2}$. By considering conservation of energy before and after the emission of the a $\gamma$-ray:

$$
E_{0}+\frac{1}{2} M V^{2}=E_{\gamma}+\frac{1}{2} M(V+v)^{2}
$$

The actual energy of the photon emitted is given by:

$$
\begin{aligned}
E_{\gamma} & =E_{0}-\frac{1}{2} M v^{2}-M v V \\
& =E_{0}-E_{R}-E_{D}
\end{aligned}
$$

The energy $E_{\gamma}$ of the emitted photon in the above equation is the excitation energy $E_{0}$ reduced by the recoil kinetic energy $E_{R}$ of the nucleus and the thermal or Doppler energy $\left(E_{D}=M v V\right)$ which is proportional to the initial velocity $V$ from the thermal motion of the nucleus. The recoil nucleus kinetic energy $E_{R}$ expressed by:

$$
E_{R}=\frac{1}{2} M v^{2}
$$

Equating the components of momentum before and after the emission process of $\gamma$-ray gives:

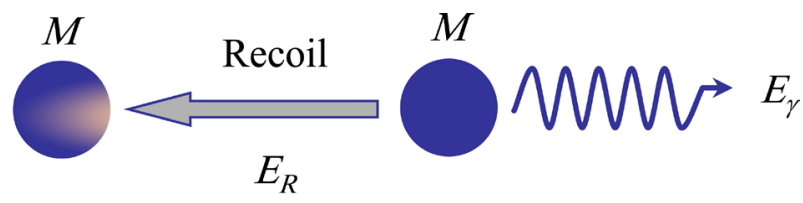

Figure 1. Recoil in a free nuclei in emission or absorption of a $\gamma$-ray. 


$$
M V=M(V+v)+\frac{E_{\gamma}}{c}
$$

where $\left(E_{\gamma} / c\right)$ is the momentum of the photon and $\mathrm{c}$ is the speed of light. The recoil momentum of the $\gamma$-ray is:

$$
M v=-\frac{E_{\gamma}}{c}
$$

Inserting Equation (5) into Equation (3), the expression for the recoil energy $E_{R}$ of the free nucleus after the emission of the $\gamma$-ray is given by:

$$
E_{R}=\frac{E_{\gamma}^{2}}{2 M c^{2}}
$$

Since the atoms of the emitter as well as the absorber have thermal energy, they move in certain velocities, and for each velocity the emitted wave's frequency is changed according to the Doppler shift. The Doppler energy $E_{D}$ is dependent on the thermal motion of the nucleus, and will therefore have a distribution of values which is temperature dependent. A mean value, $\bar{E}_{D}$, can be expressed [7] which is related to the mean kinetic energy $\bar{E}_{k}$ per translational degree of freedom of a free atom.

$$
\bar{E}_{D}=E_{\gamma} \sqrt{\frac{2 \bar{E}_{k}}{M c^{2}}} ; \quad \bar{E}_{k} \cong \frac{1}{2} k_{B} T
$$

where $k_{B}$ is Boltzmann's constant and $T$ is the absolute temperature. As a result, the statistical distribution in energy of the emitted $\gamma$-rays is displaced from the true excited state energy by $-E_{R}$ and broadened by $E_{D}$ into a Gaussian distribution of width $2 \bar{E}_{D}$. The distribution for absorption has the same shape but is displaced by $+E_{R}$. Figure 2 illustrated schematically the statistical distributions for the emitting and absorbing atoms. These energy distributions must strongly overlap in order for resonant absorption to occur.

The recoil energy is much larger than the natural linewidth $\left(\Gamma_{0} \approx 10^{-8} \mathrm{eV}\right)$ of the nuclear energy involved which is determined by the Heisenberg's uncertainty principle as $\Gamma_{0}=\hbar / \tau$, where $\hbar$ is Planck's constant and $\tau$ is the mean life time of

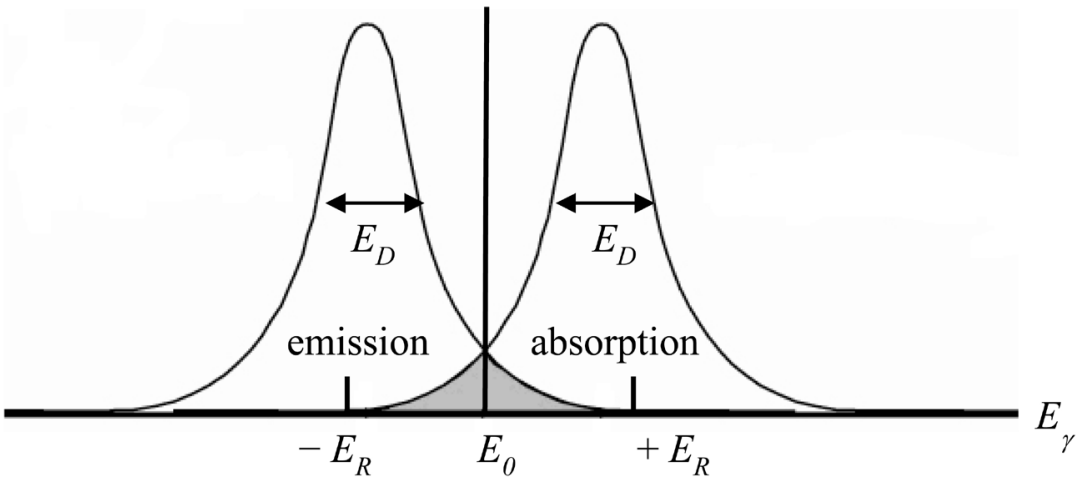

Figure 2. The statistical distribution in the $\gamma$-rays energy for the resonant overlap for successive emission and absorption. 
the excited state, e.g. for $E_{\gamma}=21.64 \mathrm{keV}$ of ${ }^{151} \mathrm{Eu}$ nucleus, $E_{R} \approx 10^{-3} \mathrm{eV}$. This results in a reduction of the $E_{\gamma}$ energy, (beyond the $\Gamma_{0}$ limit) therefore, nuclear resonant absorption of $\gamma$-ray emitted by the free nuclei can not occur.

Thus, the resonance emission and absorption can take place under the condition that $2 E_{R} \leq \Gamma_{0}$. This resonance condition is fulfilled when the recoil momentum or energy upon emission or absorption of a $\gamma$-ray is taken up by the solid as a whole rather than by a free nucleus. As the equations for recoil energy and Doppler broadening contain the reciprocal mass $M, E_{R}$ and $\bar{E}_{D}$ become very small and much lower than $\Gamma_{0}$ and thereby fulfill the resonance condition.

If the nucleus is bound in a solid, in which the movements of atoms are superpositions of quantized vibrational modes, then the recoil energy $E_{R}$ must be almost as large as the energy of the vibration quanta phonon, in order to excite a phonon. In the Einstein model of lattice vibrations, there is only one phonon frequency $\omega$. The lattice vibrations are quantized, and the recoil energy can only be transferred to the lattice if it takes place in integral multiples $\hbar \omega$. During the emission of a gamma quantum, part of the energy $E_{0}$ may be lost to the lattice by exciting a vibrational state. In this case the energy of the gamma quantum is reduced by the amount $(0, \pm \hbar \omega, \pm 2 \hbar \omega, \cdots)$ and the energy of the gamma quantum will then be too small to be resonantly absorbed by another nucleus. If the recoil energy $E_{R}<\hbar \omega$, then either zero-phonon event or one-phonon event ( $\left.\hbar \omega\right)$ of vibrational energy may be transferred to the lattice. It has been shown [8] through a theoretical consideration that when an average is taken over many emission processes, the energy transferred per event is exactly equal $E_{R}$. Using this fact we may write an expression which leads directly to the fraction, $f$, of emission events which takes place without lattice excitation (zero-phonon transition), and a fraction $(1-f)$ transfers one phonon energy $\hbar \omega$ :

$$
f=(1-f) \hbar \omega \quad \text { or } \quad f=1-\frac{E_{R}}{\hbar \omega}
$$

Only these events (i.e. recoilless fraction $f$ ) give rise to resonance emission or absorption, i.e. to the Mössbauer effect. $f$ is called Mössbauer-Lamb factor.

\subsection{The Lamb-Mössbauer Factor}

The probability of recoil-free fraction of $\gamma$-rays emitted or absorbed without loss of energy to the lattice vibrations is related to the Mössbauer-Lamb factor $f$ which can be written as [3]:

$$
f=\exp \left(-k_{\gamma}^{2}\left\langle x^{2}\right\rangle\right) ; \quad k_{\gamma}=\frac{E_{\gamma}}{\hbar c}=\frac{2 \pi}{\lambda}
$$

where $k_{\gamma}$ is the wave number of $\gamma$-ray with the wavelength of the gamma ray $(\lambda=$ $0.57 \AA$ ) in case of ${ }^{151} \mathrm{Eu}$.

$\left\langle x^{2}\right\rangle$ is the mean square amplitude for thermal vibrations of the emitting or absorbing nucleus in the direction of $\gamma$-ray. The Mössbauer Lamb factor $f$ value when it increases, leads to an increase the Mössbauer effect at relatively low $\gamma$-ray energy and at low temperature, $\left\langle x^{2}\right\rangle$ decreases with lowering tempera- 
ture. Therefore, most Mössbauer spectroscopy experiments are performed at low temperature such as liquid nitrogen or liquid helium. For the Debye model of lattice vibrations Equation (9) has the form [3]:

$$
f=\exp \left(-\frac{3 E_{R}}{2 k_{B} \Theta_{D}}\left(1+4\left(\frac{T}{\Theta_{D}}\right)^{2} \int_{0}^{\frac{\Theta_{D}}{T}} \frac{x}{\mathrm{e}^{x}-1} \mathrm{~d} x\right)\right)
$$

where $\Theta_{D}$ is the Debye temperature of the crystal in which the nucleus is bound, $T$ is the absolute temperature of the crystal at which the experiment is performed and $k_{B}$ is the Boltzmann constant. Since the fraction $f$ is strongly temperature dependent and relative to the Debye temperature, decreases as the temperature increases and conversely, an approximations can be made for very low and high temperature conditions:

$$
\begin{aligned}
& f=\exp \left(\frac{-3 E_{R}}{2 k_{B} \Theta_{D}}\right) \quad \text { for } T \ll \Theta_{D} ; \\
& f=\exp \left(\frac{-6 E_{R} T}{k_{B} \Theta_{D}^{2}}\right) \text { for } T>\Theta_{D} ;
\end{aligned}
$$

\subsection{Principle of Mössbauer Spectroscopy}

In the transmission geometry of Mössbauer experiment, a sample is exposed to a $\gamma$-ray source and a detector measures the intensity of the beam transmitted through the sample. This technique is achieved by moving a source relative to an absorber at a variable velocity. In this way, the energy of the emitted $\gamma$-rays is shifted due to the Doppler effect in order to have a resonance between a source and an absorber, the energy increases if a source moves toward an absorber and decreases when it moves away. Shifting and splitting of the nuclear energy levels between a source and an absorber can be measured at velocities in the order of $\mathrm{mm} / \mathrm{s}$. The resulting Mössbauer spectra is a plot of $\gamma$-ray transmission intensity as a function of the source velocity which can be converted to energy shift. A basic set-up for a Mössbauer experiment in transmission geometry and a typical result are shown Figure 3(a) and Figure 3(b) respectively. The Doppler energy shift $\left(E_{D}\right)$ as a function of velocity $(v)$ is equal to [3]:

$$
E_{D}=E_{\gamma} \frac{v}{c}
$$

\subsection{Gamma Ray Source}

Gamma ray is the most energetic radiation in the electromagnetic spectrum; it has the lowest wavelength and the highest frequency in the spectrum. $\gamma$-rays are sent out spontaneously by some radioactive nuclei and having energies in a range $10-100 \mathrm{keV}$. One of the most used Mössbauer isotopes among rare earth elements is ${ }^{151} \mathrm{Eu}$. The most popular source for Mössbauer spectroscopy with ${ }^{151} \mathrm{Eu}$ is ${ }^{151} \mathrm{SmF}_{3}$. Such source has a high recoil free fraction even at room temperature and a single emission line with no appreciable line broadening. The nuclear 
(a)

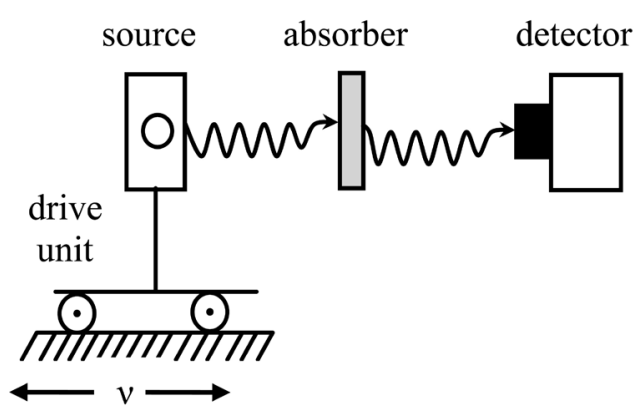

(b)

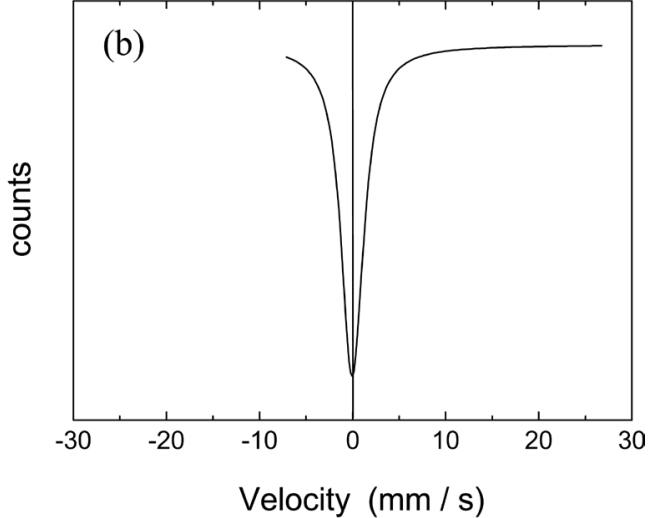

Figure 3. (a) Set-up for a Mössbauer spectroscopy experiment in transmission geometry and (b) Typical Mössbauer spectrum in the absence of splitting or shift of the nuclear levels by hyperfine interactions.

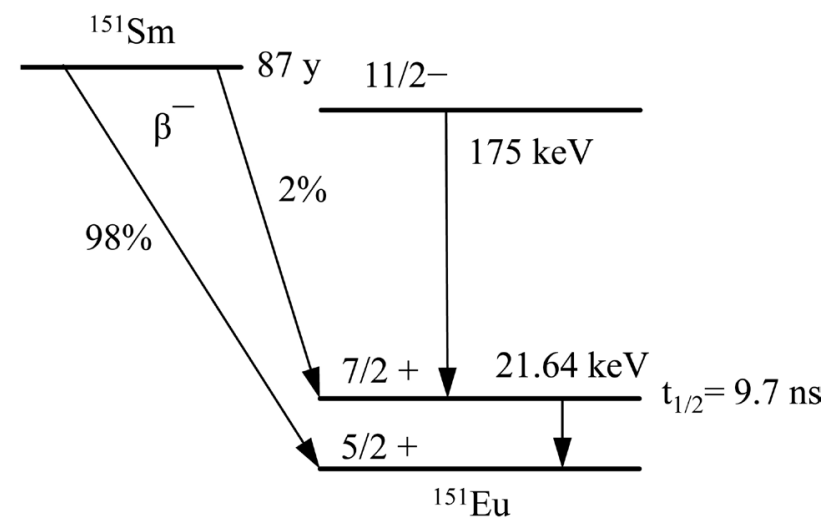

Figure 4. The nuclear decay schemes for the Mössbauer isotope ${ }^{151} \mathrm{Eu}$ [9].

decay scheme for ${ }^{151} \mathrm{Sm}, 21.64 \mathrm{keV}$ level and the value of relevant parameters are shown in Figure 4. The half-life time of ${ }^{151} \mathrm{Sm}$ is 87 years. It decays first to a metastable state of ${ }^{151} \mathrm{Eu}$ by electron capture to the excited state $I_{e}=7 / 2(21.64 \mathrm{keV})$ or to the ground state $I_{g}=5 / 2$ by the $\gamma$-ray emission. The $21.64 \mathrm{keV}$ state decays in turn to the ground state by $\gamma$-ray emission or internal conversion. The half-life of the $21.64 \mathrm{keV}$ excited state is $9.7(3) \mathrm{ns}$, giving rise to energy uncertainty. A Mössbauer $\gamma$-ray then has a natural linewidth $\left(2 \Gamma_{0}\right)$ of a resonant emission and absorption of $0.652 \mathrm{~mm} / \mathrm{s}$. Other nuclear parameters of the ${ }^{151} \mathrm{Eu}$ isotope are shown in the Table 1.

\section{Hyperfine Interactions}

The primary application of Mössbauer is to use the nucleus as a local probe when it is embedded in a solid state lattice. The interaction between a nucleus and its surrounding environment is known as a hyperfine interaction. These interactions are very small compared to the energy levels of the nucleus itself but the extreme energy resolution of the Mössbauer effect enables these interactions to be observed. The very high energy resolution of the Mössbauer spectroscopy permits small energy shifts $(\sim$ neV) to be measured. The physical reason for such an energy shift is the hyperfine interactions of a nuclear property with that of an 
Table 1. The nuclear parameters of the Mössbauer isotope ${ }^{151} \mathrm{Eu}$ [10] [11].

\begin{tabular}{ccc}
\hline Parameters & Ground State & Excited State \\
\hline Spin $I$ & $5 / 2$ & $7 / 2$ \\
Nuclear Quadrupole Nuclear Q (barns) & $1.14(5)$ & $1.50(7)$ \\
Nuclear Magnetic Moment $\left(\mu_{\mathrm{N}}\right)$ & $3.465(2)$ & $2.587(2)$ \\
Energy $E_{0}(\mathrm{keV})$ & 21.64 \\
Recoil Energy $E_{R}\left(10^{-3} \mathrm{eV}\right)$ & $1.6484(6)$ \\
Natural Isotopic Abundance $(\%)$ & 48 \\
$\Delta\left\langle r^{2}\right\rangle=\left\langle r_{e}^{2}\right\rangle-\left\langle r_{g}^{2}\right\rangle\left(10^{-3} \mathrm{fm}^{2}\right)$ & 18.5 \\
Multipolarity & \multicolumn{2}{c}{$M 1$} \\
Selection Rule & \multicolumn{2}{c}{$\Delta I=1$} \\
& \multicolumn{2}{c}{$\Delta M_{1}=0, \pm 1$} \\
\end{tabular}

electronic or atomic property of the atom. These interactions can cause degenerate nuclear excited and ground states to split or they can lead to small shifts in energy levels. Often, the linewidth of the $\gamma$-ray $10^{-8} \mathrm{eV}$ is less than the hyperfine shifts or splitting of the levels $\left(10^{-4}-10^{-7} \mathrm{eV}\right)$, which then will be reflected in the Mössbauer spectrum, partly as a splitting of the lines and partly as shifts. Three main interactions give rise to the structure of a Mössbauer spectrum. These interactions give rise to the isomer shift, electric quadrupole splitting and the magnetic hyperfine splitting. In the following sections these interactions and associated hyperfine parameters and their influence on the nuclear energy levels are discussed.

\subsection{The Electric Monopole Interaction (Isomer Shift)}

The isomer shift arises due to the non-zero volume of the nucleus and it is directly proportional to $s$-electron density at the same nucleus. This leads to an electric monopole or Coulombic interaction, which is the interaction between electrons of the atom and the nucleus. The difference in $s$-electron density of the emitting and absorbing nuclei and the difference between the radius sizes of the nucleus in the ground and excited states affect this interaction, and lead to only a shift of the nuclear energy levels Figure 5(a). In the resulting Mössbauer spectrum, such as Figure 5(b), the isomer shift is the displacement of the absorption line away from zero velocity and is observed where the Doppler velocity applied to the source correctly modifies the source's gamma-rays energy to account for the difference between the source and the absorber. This relation between charge electron density and nuclear radius is so called isomer shift $(S)$ and is given by the equation [7]:

$$
S=\frac{2 \pi}{3} Z e^{2}\left(\left|\Psi_{a}(0)\right|^{2}-\left|\Psi_{s}(0)\right|^{2}\right)\left(\left\langle r_{e}^{2}\right\rangle-\left\langle r_{g}^{2}\right\rangle\right)
$$

where $\left|\Psi_{s}(0)\right|^{2}$ and $\left|\Psi_{a}(0)\right|^{2}$ are the electron charge densities at the emitting and absorbing nuclei respectively $\left(\left|\Psi_{s}(0)\right|^{2} \neq\left|\Psi_{a}(0)\right|^{2}\right) . Z$ is the atomic num- 
ber and $e$ is the elementary electronic charge. $\left\langle r_{e}^{2}\right\rangle$ and $\left\langle r_{e}^{2}\right\rangle$ are the mean square nuclear radii of the excited and ground states respectively $\left(r_{e} \neq r_{g}\right)$. The isomer shift measurements can provide useful information on the electronic structure surrounding the nucleus and is good for probing the valence state of Mössbauer atom. As the wave function of the $s$-electrons penetrate into outer shells changes in these shells will directly alter the $s$-electron charge density at the nucleus. For example, $\mathrm{Eu}^{2+}$ and $\mathrm{Eu}^{3+}$ have electron configurations of $(4 \vec{f})$ and $\left(4 f^{f}\right)$ respectively. The $\mathrm{Eu}^{2+}$ have less $s$-electron density at the nucleus due to the greater screening effect of $p, d$, and $f$-electrons. This produces a positive isomer shift greater in $\mathrm{Eu}^{3+}$ than $\mathrm{Eu}^{2+}$. The isomer shift of Eu nuclei in compounds containing stable divalent and trivalent Eu ions falls in two well known separated regions and varies in the range between $\approx-8$ and $\approx-14 \mathrm{~mm} / \mathrm{s}$ for $\mathrm{Eu}^{2+}$ and between $\approx 0$ and $\approx+4 \mathrm{~mm} / \mathrm{s}$ for $\mathrm{Eu}^{3+}$ [12]. The ${ }^{151} \mathrm{Eu}$ Mössbauer isomer shift can be used to investigate the valence state in Eu-based compounds, due to this larger difference $(\Delta \approx 14 \mathrm{~mm} / \mathrm{s})$ between $\mathrm{Eu}^{2+}$ and $\mathrm{Eu}^{3+}$ compared to the linewidth of the resonance line $(3 \mathrm{~mm} / \mathrm{s})$.

\subsection{The Electric Quadrupole Interaction (Quadrupole Splitting)}

If the nucleus has a non-spherical charge distribution (nuclear spin quantum number $I>1 / 2$ ), then it has electric quadrupole moment [7]. Therefore, the quadrupole moment can interact with an electric field gradient (EFG) at the nucleus due to the surrounding electrons, producing a splitting in the nuclear energy levels. The electric field gradient could have two components: one due to the asymmetry of the electron charge and another to the asymmetry of the lattice charge distribution. Such interaction depends on the squared nuclear spin quantum number and lift only partially the degeneracy of the nuclear levels. The coordinate system of the electric field gradient tensor is chosen such that $\left|V_{x x}\right| \leq\left|V_{y y}\right| \leq\left|V_{z z}\right|$. The asymmetry parameter $\eta$ is generally defined as:

$$
\eta=\frac{V_{z z}-V_{y y}}{V_{z z}} ; \quad 0 \leq|\eta| \leq 1
$$

(a)
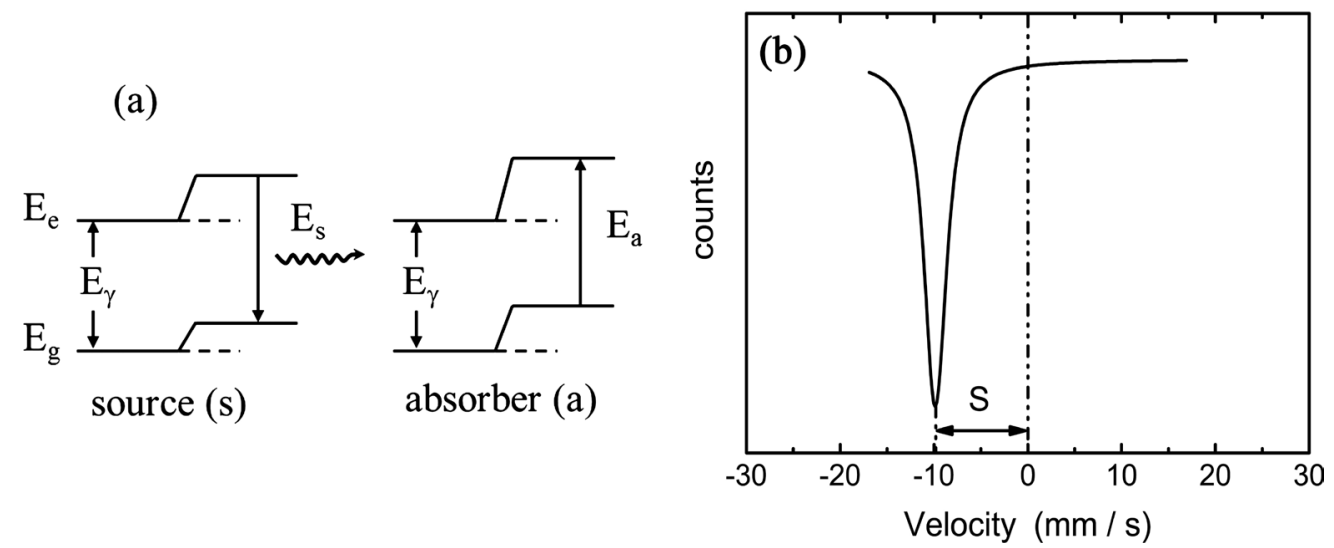

Figure 5. (a) Nuclear energy levels shifts due to electric monopole interaction and (b) Resulting Mössbauer spectrum of the isomer shift. 
The electric field gradient can be specified by two parameters $\eta$ and $V_{z z}$ The parameter $\eta$ describes deviations of the electrical field gradient from rotational symmetry around the principal component ( $\mathrm{z}$-axis). The $\mathrm{z}$-axis is often chosen to be the symmetry axis in describing a nucleus which is subjected to this hyperfine interaction, and is the axis along which the EFG is a maximum. There are two contributions to $V_{z z}[13]$ :

$$
V_{z z}=\left(1-\gamma_{\infty}\right) \cdot V_{z z}^{l a t}+(1-R) \cdot V_{z z}^{v a l}
$$

where $\gamma_{\infty}$ and $R$ are Sternheimer antishielding and shielding factors respectively. $V_{z z}^{l a t}$ is the contribution of the crystal lattice, i.e. it results from the ionic charges of the atoms which surround the Mössbauer isotope. $V_{z z}^{v a l}$ describes the contribution of the localized electron of Mössbauer atoms, due to incompletely filled electron shells (valence electrons). In $4 f$ systems this contribution is mostly dominated by a non-spherical charge distribution of the $4 f$ electrons, however in some cases, other valence electrons (e.g. $6 p$ or $5 d$ ) have a significant influence. The expression for the electric quardrupole interaction gives rise to the following energy levels scheme:

$$
E_{Q}=\frac{e Q V_{z z}}{4 I(2 I-1)}\left(3 M^{2}-I(I+1)\right)\left(1+\frac{\eta^{2}}{3}\right)^{\frac{1}{2}}
$$

with $e Q$ being the nuclear quadrupole moment and $I$ the nuclear spin of the nuclear state. $V_{z z}$ is the max value of the electrical field gradient along a symmetry axis at the nucleus. $E_{Q}$ dependent on the absolute value of the magnetic quantum number $M$ and it is degenerate in respect of the sign of $M$.

$M=-I,-I+1, \cdots, I, I_{g}=5 / 2, I_{e}=7 / 2$. It means these two states configurations have the same energy. The magnetic quantum numbers determine the number of energy levels. Therefore, they determine the number of Mössbauer lines. The excited state level spin $I_{e}=7 / 2$ is split into four $M$ sublevels $( \pm 7 / 2, \pm 5 / 2, \pm 3 / 2$, $\pm 1 / 2$ ), while the ground state level with spin $I_{g}=5 / 2$ is split into three $M$ sublevels $( \pm 5 / 2, \pm 3 / 2, \pm 1 / 2)$, as shown in Figure 6. The resultant spectrum is from transitions between ground and excited state sublevels subject to the selection rule $\Delta M=0, \pm 1$.

\subsection{Magnetic Hyperfine Field (Nuclear Zeeman Effect)}

The magnetic hyperfine splitting is due to the interaction of magnetic moments $\mu$ of the ground and excited states of the nucleus with a magnetic field with the condition that the spin quantum number $I>0$ [7]. Such interactions lift completely the degeneracy of the nuclear levels which correspond to different orientations of the spin $I$. The Hamiltonian for magnetic hyperfine dipole interaction is given as:

$$
H_{m}=-\boldsymbol{\mu} \cdot \boldsymbol{B}_{\text {eff }}=-g \mu_{N} \boldsymbol{I} \cdot \boldsymbol{B}_{\text {eff }}
$$

where $B_{\text {eff }}$ is referred to as the size of the magnetic hyperfine field at the position of the nucleus. $\left(\mu_{N}=5.050 \times 10^{-27} \mathrm{JT}^{-1}\right)$ is the nuclear magneton, and $g$ is referred 
to as the $g$-factor $\left(g=\mu / I \mu_{N}\right)$ of the nuclear state. The spin $I$ and $g$ are usually different for the excited and ground states. Due to this interaction, the magnetic field splits the nuclear energy levels of spin quantum number $I$ into $2 I+1$ equally spaced non-degenerate levels with an energy given by:

$$
E_{m}=-\frac{\mu B}{I} M=-g \mu_{N} \boldsymbol{B}_{e f f} M
$$

$M$ is the magnetic quantum numbers and can take the values $M=-I,-I+1, \cdots, I-1, I$. Transition can take place between sublevels subject to the selection rule that $\Delta M$ should change by $0, \pm 1$ in the absorption process. Figure 7 shows the splitting of the excited state $(I=7 / 2)$ and ground state $(I=$ $5 / 2$ ) nuclear levels which gives rise to very complex ${ }^{151} \mathrm{Eu}$ Mössbauer spectra with 18 allowed possible transitions but experimentally an eight-line pattern is seen

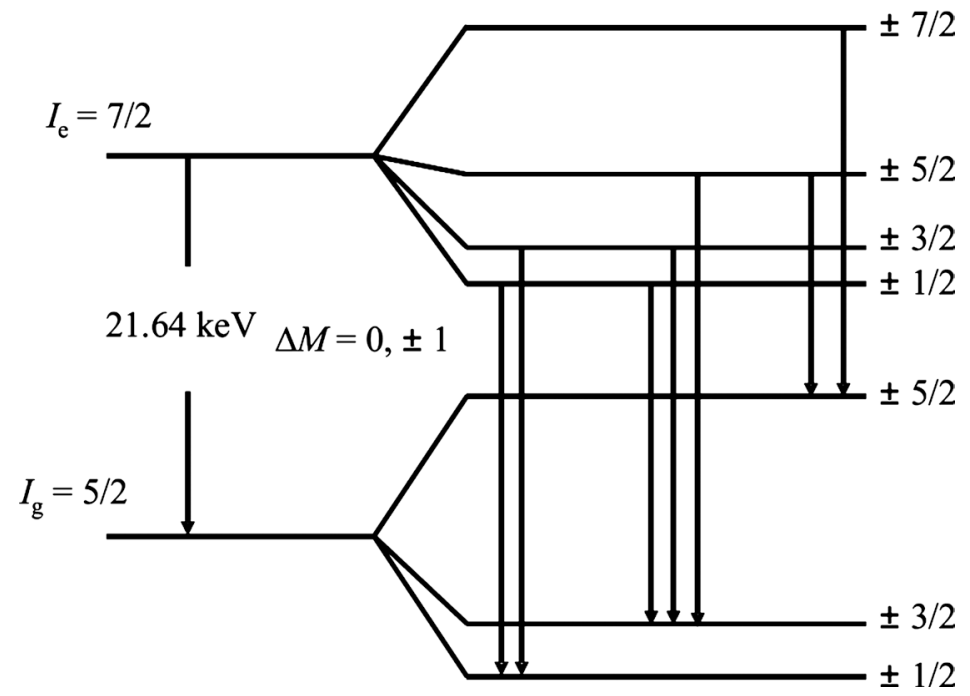

Figure 6. Nuclear energy levels of ${ }^{151} \mathrm{Eu}$ in the presence of quardrupole interaction.

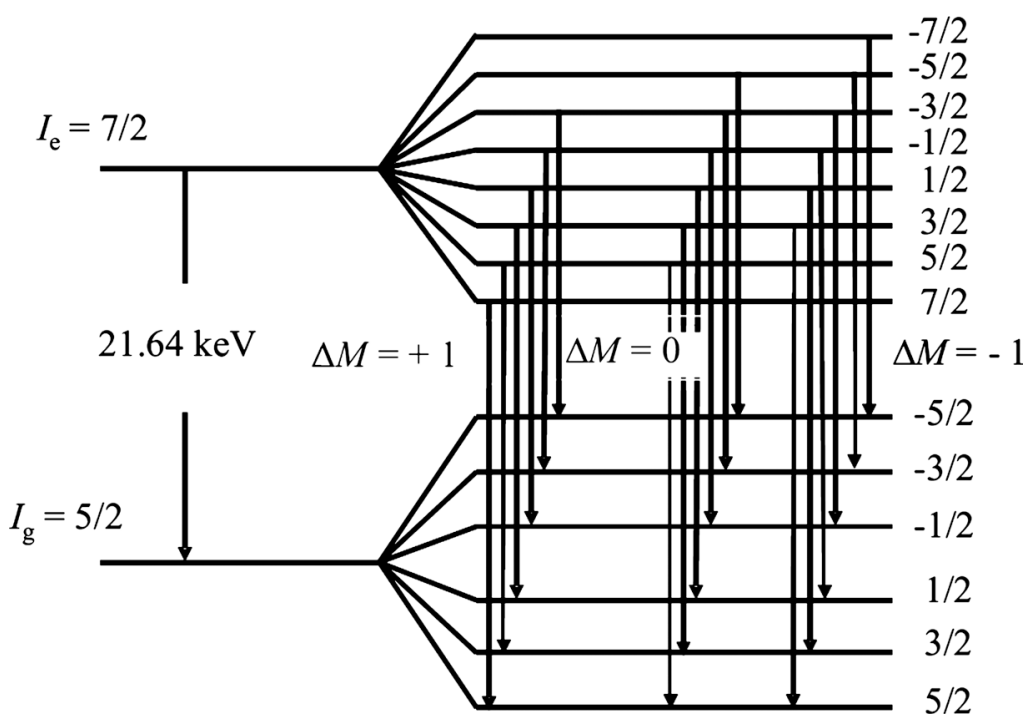

Figure 7. A schematic representation of magnetic splitting of nuclear energy levels of an ${ }^{151}$ Eu nucleus. 


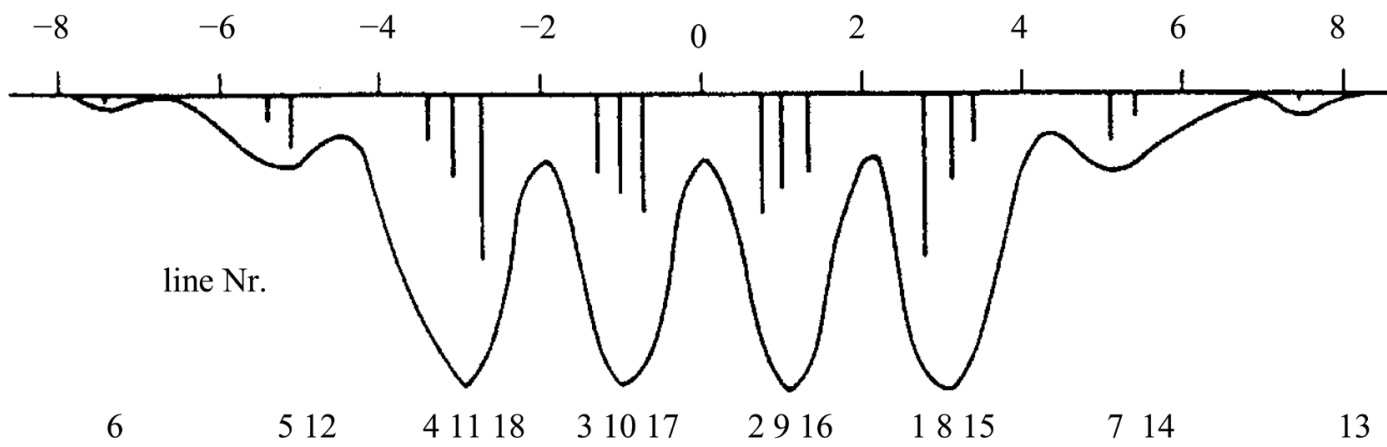

Figure 8. Resulting Mössbauer spectrum.

in the case of good counting statistics or an intense spectrum. Figure 8 shows the corresponding Mössbauer lines.

The total effective magnetic hyperfine internal field $B_{\text {eff }}$ acting on the atomic nucleus originates primarily from the atom's own electrons can be described in terms of different contributions [14]. This model has been used to analyze the different contributions to $B_{\text {eff }}$ in several Eu intermetallic compounds [15] [16].

$$
B_{e f f}=B_{c}+B_{c e}+B_{t h f}+B_{d i p}
$$

$B_{c}$ is the Fermi contact field which is caused by the core polarization of the $s$-electron density at the nucleus. It is originating from the interaction of the $S=$ $7 / 2$ spin of the $\mathrm{Eu}^{2+}$ ion with its own (nonmagnetic) electron shells [18]. It has the largest contribution to the internal field $B_{\text {eff }}$ with accepted value of $(-34 \mathrm{~T})$ [19]. In the nonrelativistic approximation, this effective field is regarded as being due to the difference of spin up and spin down densities $m(0)$ at the nucleus.

$$
B_{F}=\frac{8 \pi}{3} \mu_{B} m(0)
$$

$B_{F}$ is produced by the polarization of electrons whose wavefunctions overlap the nucleus, i.e. $s$-electrons. This polarization is due to unpaired electrons in the $f$ orbital and gives an imbalance in spin density at the nucleus from the difference in interaction between the unpaired electron with $s$-electrons of parallel or antiparallel spin to its own. This can be expressed formally as [20]:

$$
B_{F}=-\frac{8 \pi}{3} \mu_{B} \sum\left\{\left|\Psi_{\uparrow}^{s}(0)\right|^{2}-\left|\Psi_{\downarrow}^{s}(0)\right|^{2}\right\}
$$

where $\mu_{B}$ is the Bohr magneton and $\left|\Psi_{\uparrow}^{s}(0)\right|^{2}$ and $\left|\Psi_{\downarrow}^{s}(0)\right|^{2}$ are the spin up and spin down densities for $s$-electrons at the nucleus respectively. The summation is taken over all $s$-electrons. The large negative part of this field is owing to core polarization. This results from the different exchange interactions of the $1 s$, $2 s, 3 s$ and $4 s$ electrons of parallel and antiparallel spin with the $f$ shall [21]. The exchange interaction between the spin up polarized $f$ shell and the spin up $s$-electron is attractive, while that between the $f$ shell and a spin down $s$-electron is repulsive. As a result the radial parts of the two $s$-electron wave functions will be different, one being pushed toward the nucleus, the other pulled outward. Therefore, the spin densities at the nucleus no longer cancel and a Fermi contact 
interaction is observed [5].

$B_{c e}$ is the contribution from the conduction electron spin polarization produced by the $4 f$ electron of the magnetic ion itself.

$B_{t h f}$ is the transferred hyperfine field produced at an Eu nucleus by the spin polarization of the conduction electrons by the $4 f$ magnetic moment of the neighboring atoms. It has been found the transferred hyperfine fields are relatively small in Eu-intermetallic compounds [15] [16] [17]. However, $\left|B_{\text {thf }}\right|$ is very sensitive to external pressure and determine the pressure dependent of $\left|B_{\text {eff }}\right|$ [14].

$B_{d i p}$ is contribution from the dipole interaction with the moment of the electron spin. The total $B_{\text {dip }}$ dipolar field is the sum of the Lorentz field, the demagnetization field, and the contribution from neighboring magnetic dipoles; $B_{d i p}$ is usually very small [22] compared to the other contributions.

\section{Valence Fluctuations in Eu Intermetallic Compounds}

The phenomena of valence fluctuations has been observed in certain $4 f$ systems, such as $\mathrm{Ce}, \mathrm{Pr}, \mathrm{Sm}, \mathrm{Eu}$ and $\mathrm{Yb}$ compounds. This is due to the fact that the respective rare earth ions can in principle exist in two different valence states with different numbers of $4 f$ electrons (see Figure 9). Temperature and pressure variations, or alloying with proper elements, may lead to a quasidegeneration of the two valence states, giving rise to a nonintegral average occupation number of the $4 f$ shell (i.e. intermediate valence state). The transition from stable (normal) into the intermediate valence state is accompanied by anomalous changes of the physical properties of the $4 f$-system. An overview on the intermediate valence phenomena and their theoretical treatments have been given by [23] [24] [25].

In this investigation, we focus on the description of the phenomena of magnetically ordered $\mathrm{Eu}$ intermetallic compounds systems. Europium ions are usually found to be either divalent $\mathrm{Eu}^{2+}$ state or nonmagnetic trivalent $\mathrm{Eu}^{3+}$ state

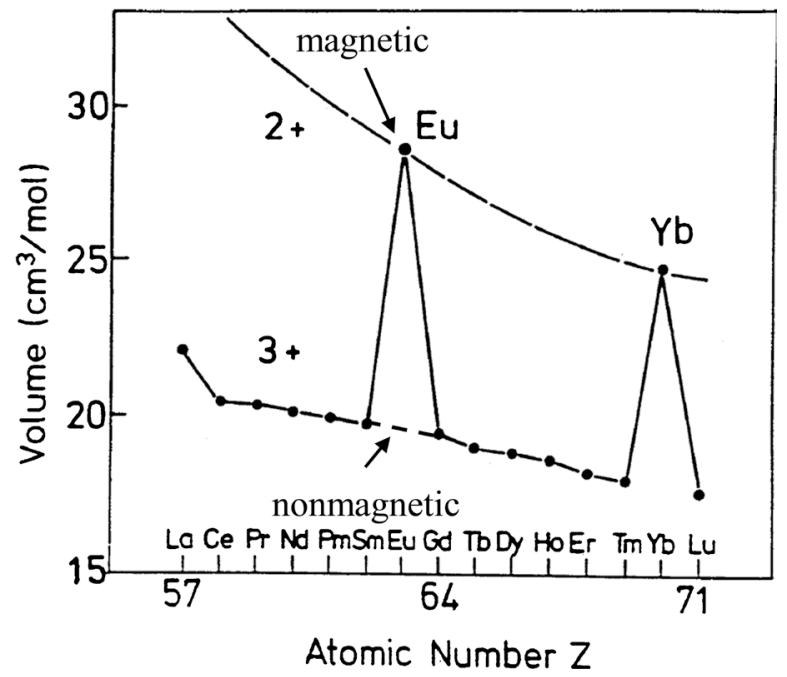

Figure 9. Volume vs. atomic number in rare earth elements. Reconstructed from reference [26]. 
in metallic compounds. Figure 9 shows the unit cell volume of rare earth elements [26]. The magnetic properties in the two valence states are quite different. The ground state configuration of the $\mathrm{Eu}^{2+}$ is $4 \bar{f}$ with ionic ground state ${ }^{8} S_{7 / 2}$, with localized magnetic moment of $7 \mu_{B}$. In the $\mathrm{Eu}^{3+}$ the configuration is $4 f^{6}$ with ionic ground state ${ }^{7} F_{0}$. In the stable compounds, the $\mathrm{Eu}^{2+}$ state is generally located well below the Fermi energy $E_{F}$. The intermediate valence (IV) case is encountered in compounds where the excitation energy $E_{\text {exc }}$ (the distance between the Fermi level $E_{F}$ and localized $4 f$ level) is relatively small $E_{e x c} \leq 300 \mathrm{~K}$, Figure 10. In such compounds the Eu ions exhibit an IV state and undergo a valence change if one changes either temperature or pressure as it has been observed, e.g. in $\mathrm{EuPd}_{2} \mathrm{Si}_{2}$ [27] [28] [29] and $\mathrm{EuCu}_{2} \mathrm{Si}_{2}$ [30] [31]. Other example of different rare earths are $\mathrm{YbCu}_{2} \mathrm{Si}_{2}[32]$ and $\mathrm{CePd}_{3}[33]$.

There are varieties of techniques to measure the mean valence in an intermediate valence system. Measurements of macroscopic properties such as magnetic susceptibility and electrical transport are indirectly affected by the existence of the valence fluctuations of the system [34] [35] [36]. On the other hand, measurements of microscopic properties by, e.g. Mössbauer effect, photoelectric emission, nuclear resonant scattering and x-ray absorption techniques provide direct information about the valence fluctuations. The difference between these techniques is set by the timescale to which they are sensitive. Techniques with a timescale that is short with respect to that of the valence fluctuation $\left(\approx 10^{-13} \mathrm{sec}\right)$ [37], are sensitive to the momentary values. Consequently, the measured spectrum will be a superposition of the spectra corresponding to both discrete valence states. This is the case in, e.g., $\mathrm{L}_{I I I} \mathrm{x}$-ray absorption edge studies. The techniques with a timescale that is much longer than of the valence fluctuations, such as Mössbauer spectroscopy the measured spectrum gives an average value for the valence.
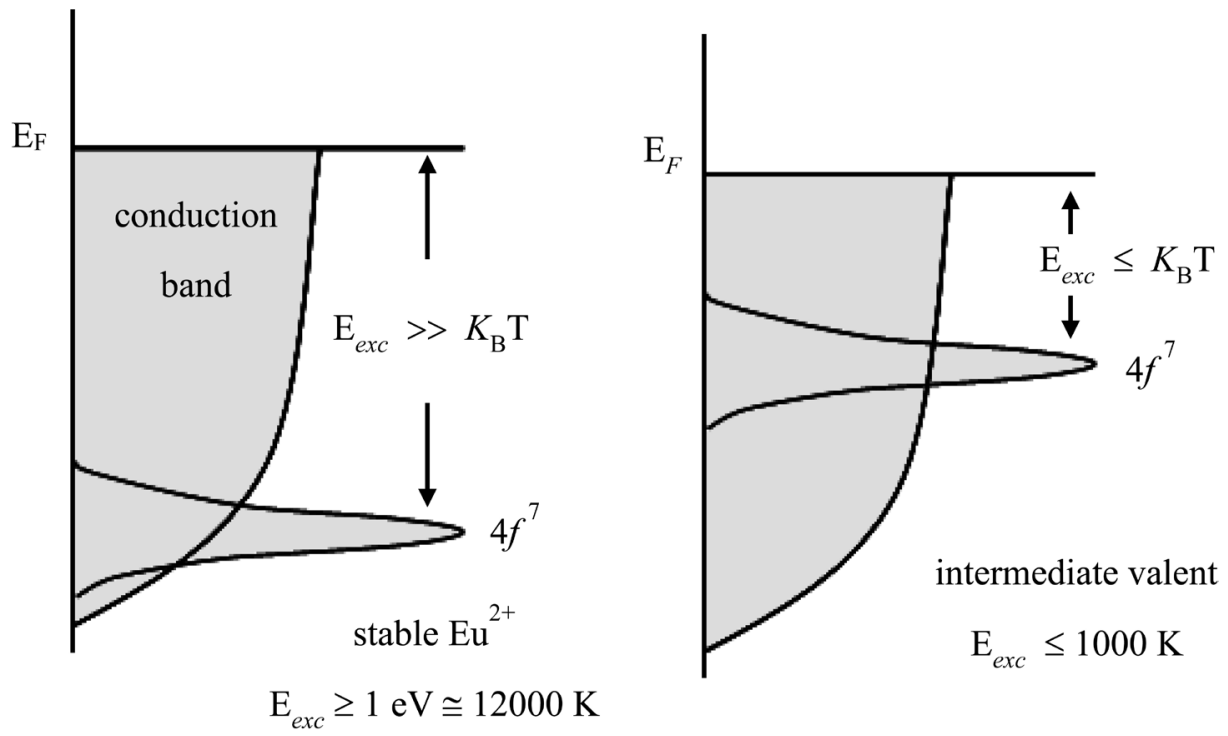

Figure 10. Schematic representation of the position of the $4 \vec{f}$ localized magnetic level relative to the Fermi level $E_{F}$, in stable and intermediate valent systems. 


\section{Ionic Model of Valence Fluctuations}

The isomer shift (S) deduced from ${ }^{151} \mathrm{Eu}$ Mössbauer spectroscopy plays important role in the field of intermediate valence Eu compounds due to the large difference in its values between the $4 \vec{f}\left(\mathrm{Eu}^{2+}\right)$ and $4 f^{\beta}\left(\mathrm{Eu}^{3+}\right)$ configurations. In the frame of the ionic the interconfigurational fluctuation (IFC) model [34] it is assumed that the valence of the Eu ions fluctuates rapidly between, the localized $4 f$ level and the conduction band, i.e. fluctuates between the integral values $2<v<$ 3 on a time scale in the order of $10^{-13} \mathrm{sec}$ to $10^{-15} \mathrm{sec}$ [38] [39]. The two configurations are separated by an excitation energy $\left(E_{\text {exc }}\right)$ which is the distance between the Fermi level $E_{F}$ and localized $4 f$ level. In this frame, the measured isomer shift $S(T, V)$ of the Eu ion is converted to the Eu mean valence $v$ through the formula [31] [39].

$$
S(T, V)=S_{2}(T, V)+S\left(S_{3}-S_{2}\right) \cdot \Delta v(T, V)
$$

The mean valence, $v$ is given by:

$$
v=2+\Delta v(T, V)
$$

where $S(T, V)$ is the measured value of the isomer shift at $4.2 \mathrm{~K}, S_{2}(T, V)$ is the stable isomer shift corresponding to the divalent state. $S_{2}$ and $S_{3}$ are the integral values of the isomer shift of $\mathrm{Eu}^{2+}$ and $\mathrm{Eu}^{3+}$ respectively. $\Delta v(T, V)$ is the occupation probability of the $\mathrm{Eu}^{3+}$ state, given the mean valence $v$ and be calculated from the measured values of $S$ at different concentrations and temperatures with suitable choice of the values for $S_{2}$ and $S_{3}$. Using the following relation [40], the value of $\Delta v$ can be used to evaluate the corresponding values of $E_{e x c}$ at $300 \mathrm{~K}$.

$$
\Delta v(T, V)=\frac{8 \exp \left[-E_{\text {exc }}(T, V) / K_{B} T^{*}\right]}{1+3 \exp \left[-480 / T^{*}\right]+5 \exp \left[-1330 / T^{*}\right]+\cdots}
$$

where $T^{*}=\sqrt{T^{2}+T_{f}^{2}}$ is the effective temperature, and $T_{f}$ is being the valence fluctuation temperature, introducing phenomenologically the width of $E_{\text {exc }}$ i.e. $T_{f}$ is related to the hybridization energy of the $4 f$ state with the conduction band state, and should tend to zero for $E_{\text {exc }} \rightarrow \pm \infty$ [30]. The multiplicity of the $\mathrm{Eu}^{2+}$ ground state $(J=7 / 2)$ is 8 , and the ground state $\mathrm{Eu}^{3+}(J=0)$ is 1 . The first and second excited states of $\mathrm{Eu}^{3+}(J=1$ and $J=2$, respectively) are located at energies of $480 \mathrm{~K}$ and $1330 \mathrm{~K}$, respectively [41].

\section{Conclusion}

The goal of this review is to create an accessible summary and familiarize the reader who is not an active practitioner of Mössbauer spectroscopy with the basic concepts of the method. First, the theory behind the Mössbauer effect is explained and the key to the success of the technique is the discovery of recoilless gamma ray emission and absorption. It is intended that this information should be sufficient for the reader to understand the application of Mössbauer spectroscopy to analysis the studied system. This paper also presents some ideas based on the nuclear physics hyperfine interactions parameters that can by de- 
rived from the Mossbauer effect. These interactions are very small compared to the energy levels of the nucleus itself but the extreme energy resolution of the Mössbauer effect enables these interactions to be observed. The very high energy resolution of the Mössbauer spectroscopy permits small energy shifts $(\sim$ neV) to be measured. Therefore, for further studies using Mössbauer spectroscopy technique with external parameters such as temperature and pressure can very precise information about valence state of Mössbauer atom, structural electronic and magnetic properties of the studied material. One could gain information at a microscopic level about valence fluctuations in Eu systems via the ${ }^{151} \mathrm{Eu}$ Mössbauer isomer shift $S$. Also, could gain a microscope insight into the change of the magnetic moment of the Eu ions via the effective magnetic hyperfine field $B_{\text {eff }}$ at the Eu nucleus. Finally, references of books and articles are given for further and more detailed information.

\section{Conflicts of Interest}

The author declares no conflicts of interest regarding the publication of this paper.

\section{References}

[1] Mössbauer, R.L. (1958) Kernresonanzfluoreszenz von Gammastrahlung in $\operatorname{Ir}^{191}$. Zeitschrift für Physik, 151, 124. https://doi.org/10.1007/BF01344210

[2] Mössbauer, R.L. (1959) Kernresonanzabsorption von y-Strahlung in $\operatorname{Ir}^{191}$. Zeitschrift für Naturforschung, 14a, 211.

[3] Frauenfelder, H. (1962) The Mössbauer Effect. Benjamin, New York.

[4] Dirken, M.W. (1963) A ${ }^{151}$ Gd Mössbauer Study on the Origin of the Electric Field Gradient.

[5] Wertheim, G.K. (1964) Mössbauer Effect: Principles and Applications. Academic Press Inc., New York.

[6] Greenwood, N.N. and Gibb, T.C. (1971) Mössbauer Spectroscopy. Chapman and Hall Ltd., London. https://doi.org/10.1007/978-94-009-5697-1

[7] Gibb, T.C. (1971) Principles of Mössbauer Spectroscopy. Chapman and Hall Ltd., London.

[8] Lipkin, H.J. (1960) Some Simple Features of the Mössbauer Effect. Annals of Physics, 9, 332-339. https://doi.org/10.1016/0003-4916(60)90035-X

[9] Stevens, J.G. (1975) Mössbauer Effect Data Index 1974. Plenum, New York, 204.

[10] Bauminger, E.R., Kalvius, G.M. and Nowik, I. (1978) Mössbauer Isomer Shifts. In: Shenoy, G.K. and Wagner, F.E., Eds., North-Holland, Amsterdam, 661.

[11] Mössbauer Effect Data Center, University of North Carolina.

[12] de Veries, J.W.C., Thiel, R.C. and Buschow, K.H.J. (1983) ${ }^{151} \mathrm{Eu}$ Isomer Shifts and Charge Transfer in Eu-Base Intermetallic Compounds. Physica, 121B, 100.

[13] Ofer, S., Nowik, I. and Cohen, S.G. (1968) Chemical Applications of Mössbauer-Spectroscopy. In: Goldanskii, V.I. and Herber, R.H., Eds., Academic Press, New York, 427.

[14] Abd-Elmeguid, M.M., Micklitz, H. and Kaindl, G. (1981) Volume Dependence of Magnetic Hyperfine Fields in Eu-Intermetallic Compounds. Physical Review B, 23, 
75. https://doi.org/10.1103/PhysRevB.23.75

[15] Nowik, I., Dunlap, B.D. and Wernik, J.H. (1973) Contributions to the Hyperfine Field in Europium Intermetallics. Physical Review B, 8, 238. https://doi.org/10.1103/PhysRevB.8.238

[16] Leowenhaupt, M. (1973) Mössbauereffekt- und Magnetisierungsmessungen an $\mathrm{Eu}_{x} \mathrm{Yb}_{1-x} \mathrm{Sn}_{3}$. Zeitschrift für Physik A: Hadrons and Nuclei, 258, 209-222. https://doi.org/10.1007/BF01392831

[17] Kropp, K., Zipf, W., Dormann, E. and Buschow, K.J.H. (1979) Indirect Exchange in Intermetallic Europium Compounds. Journal of Magnetism and Magnetic Materials, 13, 224-230. https://doi.org/10.1016/0304-8853(79)90075-1

[18] Wortmann, G. and Moser, J. (1981) Transferred Hyperfine Fields at ${ }^{119} \mathrm{Sn}$ and ${ }^{151} \mathrm{Eu}$ in $\left(\mathrm{Eu}_{0.99} \mathrm{Sn}_{0.01}\right)$ Se. Hyperfine Interactions, 10, 879-885. https://doi.org/10.1007/BF01022026

[19] Baker, J.M. and Williams, F.I.B. (1962) Electron Nuclear Double Resonance of the Divalent Europium Ion. Proceedings of the Royal Society of London Series A, 267, 283-294.

[20] Goldanskii, V.I. and Makarov, E.F. (1968) In: Goldanskii, V.I. and Herber, R.H., Eds., Chemical Applications of Mössbauer-Spectroscopy, Academic Press, New York, 24.

[21] Watson, R.E. and Freeman, A.J. (1961) Origin of Effective Fields in Magnetic Materials. Physical Review, 123, 2027-2047. https://doi.org/10.1103/PhysRev.123.2027

[22] Barb, D. (1980) Grundlagen und Anwendungen der Mössbauerspektroskopie. Akademie-Verlag, Berlin.

[23] Lawrence, J.M., Riseborough, P.S. and Parks, R.D. (1981) Valence Fluctuation Phenomena. Reports on Progress in Physics, 44, 1.

https://doi.org/10.1088/0034-4885/44/1/001

[24] Parks, R.D. (1985) Mixed Valence Phenomena: An Overview. Hyperfine Interactions, 25, 565-581. https://doi.org/10.1007/BF02354667

[25] Nowik, I. (1983) Mössbauer Studies of Valence Fluctuations. Hyperfine Interactions, 13, 89-118. https://doi.org/10.1007/BF01027247

[26] Brooks, M.S.S., Johansson, B. and Skriver, H.L. (1984) In: Freeman, A.J. and Lander, G.H., Eds., Handbook on the Physics and Chemistry of the Actinides, North-Holland, Amsterdam, Vol. 1, 153.

[27] Sampathkumaran, E.V., Gupta, L.C., Vijayaraghavan, R., Pillay, R.G., Gopalakrishnan, K.V. and Devarem H.G. (1981) A New and Unique Eu-Based Mixed Valence System: $\mathrm{EuPd}_{2} \mathrm{Si}_{2}$. Journal of Physics C: Solid State Physics, 14, L237. https://doi.org/10.1088/0022-3719/14/9/006

[28] Croft, M., Hodges, J.A., Kemly, E., Krishnan, A., Murgai, V. and Gupta, L.C. (1982) Cooperative Configuration Change in $\mathrm{EuPd}_{2} \mathrm{Si}_{2}$. Physical Review Letters, 48, 826 829. https://doi.org/10.1103/PhysRevLett.48.826

[29] Stöber, D., Saner, C., Zinn, W. and Abd-Elmeguid, M.M. (1987) Magnetic Order versus Intermediate Valence in $\mathrm{Eu}\left(\mathrm{Pd}_{1-x} \mathrm{Au}_{x}\right)_{2} \mathrm{Si}_{2}$ under High Pressure. Journal of Magnetism and Magnetic Materials, 69, 144-148.

https://doi.org/10.1016/0304-8853(87)90110-7

[30] Röhler, J., Wohlleben, D., Kaindl, G. and Balster, H. (1982) Energy Balance of Mixed-Valent Eu Ions. Physical Review Letters, 49, 65-68. https://doi.org/10.1103/PhysRevLett.49.65

[31] Abd-Elmeguid, M.M., Sauer, C. and Zinn, W. (1985) Pressure-Induced Valence 
Change of $\mathrm{Eu}$ in $\mathrm{Eu}\left(\mathrm{Pd}_{0.8} \mathrm{Au}_{0.2}\right)_{2} \mathrm{Si}_{2}$ : Collapse of Magnetic Order. Physical Review Letters, 55, 2467-2470. https://doi.org/10.1103/PhysRevLett.55.2467

[32] Sales, B.C. and Viswanathan, R. (1976) Demagnetization due to Interconfiguration Fluctuations in the $\mathrm{RE}-\mathrm{Cu}_{2} \mathrm{Si}_{2}$ Compounds. Journal of Low Temperature Phystcs, 23, 449-467. https://doi.org/10.1007/BF00116933

[33] Croft, M., Neifeld, R., Segre, C.U., Raaen, S. and Parkss, R.D. (1984) Ce Valence Variation in Intermetallic Alloys: $L_{I I I}$ Absorption Spectroscopy Results. Physical Review $B, 30,4164-4169$. https://doi.org/10.1103/PhysRevB.30.4164

[34] Wohlleben, D.K. (1981) In: Falicov, L.M., Hanke, W. and Maple, M.B., Eds., Valence Fluctuations in Solids, North-Holland, Amsterdam, 1.

[35] Hossain, Z., Geibel, C., Senthilkumaran, N., Deppe, M., Baenitz, M., Schiller, F. and Molodtsov, S.L. (2004) Antiferromagnetism, Valence Fluctuation, and Heavy-Fermion Behavior in $\mathrm{EuCu}_{2}\left(\mathrm{Ge}_{1-x} \mathrm{Si}_{x}\right)_{2}$. Physical Review B, 69, Acticle ID: 014422. https://doi.org/10.1103/PhysRevB.69.014422

[36] Fukuda, S., Nakanuma, Y., Sakurai, J., Mitsuda, A., Isikawa, Y., Ishikawa, F., Goto, T. and Yamamoto, T. (2003) Application of Doniach Diagram on Valence Transition in $\mathrm{EuCu}_{2}\left(\mathrm{Si}_{x} \mathrm{Ge}_{1-x}\right)_{2}$. Journal of the Physical Society of Japan, 72, 3189-3196. https://doi.org/10.1143/JPSJ.72.3189

[37] Nagarajan, R., Sampathkumaran, E.V., Gupta, L.C., Vijayaraghavan, R. and Padalia, B.D. (1981) X-Ray Absorption Spectroscopic Study of a Mixed Valence System, $\mathrm{EuPd}_{2} \mathrm{Si}_{2}$. Physics Letters A, 81, 397-398.

https://doi.org/10.1016/0375-9601(81)90100-6

[38] Hirst, L.L. (1971) Interactions between a $3 d$ Impurity and Conduction Electrons. Zeitschrift für Physik A: Hadrons and Nuclei, 244, 230-244. https://doi.org/10.1007/BF01395568

[39] Bauminger, E.R., Froindlich, D., Nowik, I., Ofer, S., Felner, I. and Mayer, I. (1973) Charge Fluctuations in Europium in Metallic $\mathrm{EuCu}_{2} \mathrm{Si}_{2}$. Physical Review Letters, 30, 1053-1056. https://doi.org/10.1103/PhysRevLett.30.1053

[40] Bauminger, E.R., Felner, I., Froindlich, D., Levron, D., Nowik, I., Ofer, S. and Yanovsky, R. (1974) Mössbauer Effect Studies of Interconfiguration Fluctuations in Metallic Rare Earth Compounds. Journal de Physique Colloques, 35, C6.

[41] Ksenofontov, V., Kandpal, H.C., Ensling, J., Waldeck, M., Johrendt, D., Mewis, A., Gütlich, P. and Felser, C. (2006) Verwey-Type Transition in EuNiP. Europhysics Letters, 74, 672-678. https://doi.org/10.1209/epl/i2005-10563-6 